\title{
Visual assessment of right ventricular function by echocardiography: how good are we?
}

\author{
Matthias Schneider ${ }^{1}\left[\right.$ - Hong Ran $^{2} \cdot$ Stefan Aschauer ${ }^{1} \cdot$ Christina Binder $^{1} \cdot$ Julia Mascherbauer $^{1} \cdot$ Irene Lang $^{1}$. \\ Christian Hengstenberg ${ }^{1} \cdot$ Georg Goliasch $^{1} \cdot$ Thomas Binder $^{1}$
}

Received: 30 April 2019 / Accepted: 17 June 2019 / Published online: 24 June 2019

(c) The Author(s) 2019

\begin{abstract}
The complex anatomy and physiology of the right ventricle (RV) is a major limitation of visual echocardiographic gradation of RV systolic function (RVF). The aim of this study was to compare visual assessment ("eyeballing") of RVF with gold standard magnetic resonance imaging (MRI)-derived right ventricular ejection fraction (RVEF). Medical professionals from a range of clinical settings and with varying degrees of echocardiography experience were recruited via an online ultrasound teaching platform. In an anonymized web-based test, participants graded RVF in 10 patients with varying degrees of RVF via "eyeballing" of an RV-focused four-chamber view. Two skills were evaluated: (1) ability to differentiate between normal and reduced RVF; and (2) ability to determine the correct degree of RV systolic dysfunction. A total of 868 participants from 99 countries were included. For detection of reduced RVF (MRI-RVEF < 50\%), sensitivity was 97.1\%, 96,8\%, 96.5\%, and $95.8 \%$ and specificity was $55.7 \%, 52.8 \%, 54.6 \%$, and $42.5 \%$ for the expert, advanced, intermediate, and beginner groups, respectively. For determination of the correct degree of RV dysfunction, even experienced examiners assigned a diagnosis that was discordant with MRI in $>40 \%$ of cases. In the present cohort, "eyeballing" was associated with excellent sensitivity but poor specificity in terms of differentiation between normal and abnormal RVF. Even among experts, classification of the degree of RV dysfunction was imprecise. In accordance with current guidelines, the present data suggest that "eyeballing" should be combined with evaluation of other echocardiographic parameters of RVF.
\end{abstract}

Keywords Transthoracic echocardiography $\cdot$ Right ventricular function · Visual assessment $\cdot$ Eyeballing

\section{Background}

Evaluation of right ventricular function (RVF) is an essential component of echocardiographic evaluation. While the geometry of the left ventricle allows precise evaluation of ejection fraction via visual assessment (“eyeballing") [1, 2], determination of the degree of RVF is rendered challenging by the complex anatomy and physiology of the right ventricle (RV).

Current guidelines for echocardiographic chamber quantification recommend that RVF should be evaluated by both visual assessment and measurement of at least one additional

Matthias Schneider

matthias.schneider@meduniwien.ac.at

1 Department of Internal Medicine II, Medical University of Vienna, Waehringer Guertel 18-20, 1090 Wien, Austria

2 Department of Echocardiography, Nanjing First Hospital, Nanjing Medical University, Nanjing, China
RVF parameter, such as tricuspid annular plane systolic excursion (TAPSE), tissue Doppler imaging of the basal free lateral wall of the RV ( $S^{\prime}$ ), or fractional area change (FAC) [3, 4]. These conventional echocardiographic parameters show good correlation with the corresponding cardiac magnetic resonance imaging (CMR) measurements [5]. However, for many of these parameters, echocardiographic evaluation is complex and time consuming. Unsurprisingly, a recent study by the present authors showed that "eyeballing" is the most widely used method for the echocardiographic classification of RVF [6].

Previous studies have investigated the reliability of RVF assessment using "eyeballing" alone. However, the results have been inconsistent. While two studies suggested that "eyeballing" alone is insufficient in terms of determining the degree of RVF [7, 8], a recent study concluded that RVF can be determined reliably by "eyeballing" alone if the examiner is very experienced [9]. These previous studies were performed at single centers and included small numbers of 
examiners and patients. International data on the use of "eyeballing" alone to assess RVF are therefore lacking.

In recent years, rapid developments in ultrasound technology and the increasingly widespread availability of wireless handheld devices have rendered RV imaging possibilities beyond the context of highly specialized cardiology centers. In the non-cardiology setting, emergency cardiac ultrasound protocols include a quick visual assessment of right and left ventricular function. Since further dissemination of emergency cardiac ultrasound is anticipated, in the foreseeable future RVF will be assessed by more, and inevitably less experienced, examiners. Evaluation of the accuracy of "eyeballing" is therefore crucial in terms of patient safety.

The aims of the present study were twofold. First, to evaluate the ability to differentiate between normal and reduced RVF by "eyeballing". Second, to assess how visual gradation into class of dysfunction compares to the gold standard CMR-derived right ventricular ejection fraction (RVEF).

\section{Methods}

Participants were recruited via the network of the Englishlanguage based online ultrasound teaching platform 123 sonography (https://www.123sonography.com). All participants completed an online questionnaire and a web-based test. The study was open for participation between April 1 and July 31, 2017. The study was approved by the ethics committee of the University of Vienna (EK \#1288/2016). The study protocol conformed to the ethical guidelines of the Declaration of Helsinki.

\section{Questionnaire}

No data were collected concerning the names of the participants or their respective institutions. Baseline demographic data were collected, such as age, country in which the participant was employed, profession, and work setting (e.g., university hospital, private practice). To establish study groups, the participants were asked to specify their level of echocardiography experience: beginner, intermediate, advanced, or expert.

\section{Web-based test}

The RV focused apical four-chamber view video loop of 10 real patients was presented online to the study participants. The participants were instructed to grade each RV solely by "eyeballing" according to a four-grade scale (normal, mildly reduced RVF, moderately reduced RVF, severely reduced RVF).

\section{Selection of patient data}

The ten patients were selected retrospectively from the echocardiography database of the University Hospital of Vienna. Inclusion criteria were good echocardiographic image quality and a CMR within three months of the echocardiographic examination. Selected patients represented the entire RVF spectrum from normal to severely reduced systolic function. The clinical, CMR, and echocardiographic characteristics of all 10 patients are summarized in Table 1 .

\section{Echocardiography}

Standard transthoracic echocardiograms (2D, Doppler) were obtained from all 10 patients using echocardiography systems equipped with $3.5 \mathrm{MHz}$ transducers (Vivid E9; General Electric Healthcare). Echocardiography was performed in accordance with the recommendations of the American Society of Echocardiography and the European Association of Cardiovascular Imaging [3, 4]. From the stored echo loops of each patient, a high-quality RV-focused apical fourchamber view was selected. In all 10 patients, the following measures were obtained: TAPSE; FAC; global longitudinal strain of the free lateral wall of the RV (GLS-RV); and S'. Figure 1 shows the cut-offs that were used to define reduced RVF [4].

\section{Cardiac magnetic resonance imaging}

All cardiac CMR examinations were performed by using a 1.5-T imager (Magnetom Avanto; Siemens Medical Solutions, Erlangen, Germany) with standard protocols. Steadystate images were used for cine imaging (repetition time msec/echo time msec, $3.2 / 1.2$; ip angle, $64^{\circ}$; voxel size, $1.431 .436 \mathrm{~mm} ; 1,803,256$ matrix). A transaxial stack of images was used for volumetric assessment of RVs. Trabeculations, papillary muscles, and the right ventricular outflow tract were included as part of the RV volume as previously suggested [10]. RV dysfunction was defined as $\mathrm{RV}$ ejection fraction $<50 \%$ and was further subdivided into mildly decreased (RV ejection fraction 40-49\%), moderately decreased (RV ejection fraction 30-39\%) and severely decreased (RV ejection fraction <30\%) [11]. Two independent observers (SA, JM) blinded to clinical data read all CMR studies.

All patients had undergone both CMR and an echocardiographic examination within a mean period of $12 \pm 22$ days. According to CMR, five (50\%) patients had normal RVEF (RVEF > 50\%), one (10\%) patient had mildly reduced RVEF (RVEF: $40-50 \%$ ), three (30\%) patients had moderately reduced RVEF (RVEF: 30-40\%), and one (10\%) patient had 


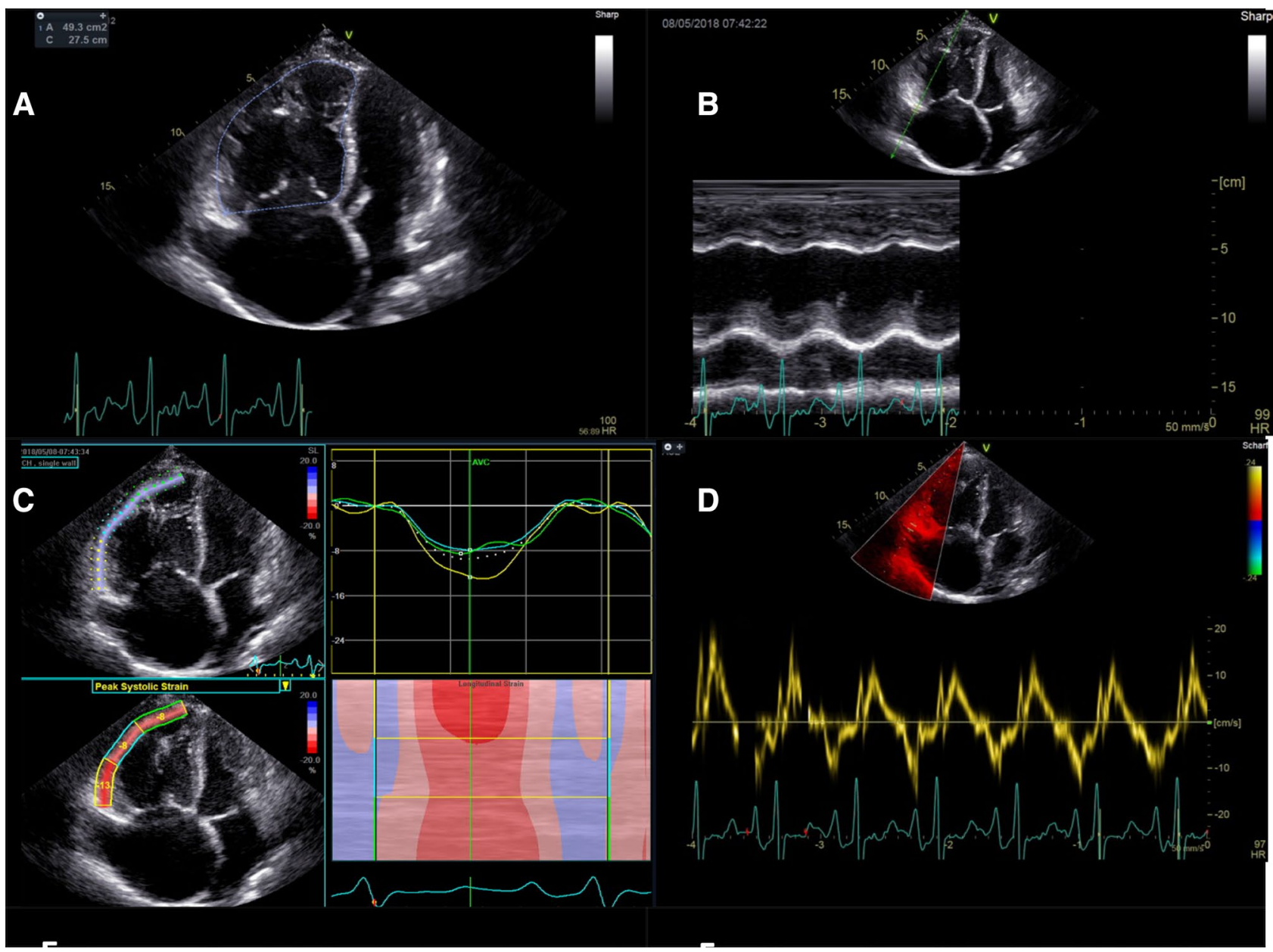

Fig. 1 Evaluation of right ventricular function: RV end-diastolic for calculation of right ventricular fractional area change $(\mathbf{a}, \mathrm{RVF}$ reduced if $<35 \%$ ), TAPSE (b, RVF reduced if $<17 \mathrm{~mm}$ ), longitudinal strain of the free lateral wall of the right ventricle $(\mathbf{c}$, RVF reduced if $>-20 \%$ ), and $\mathrm{S}^{\prime}(\mathbf{d}, \mathrm{RVF}$ reduced if $<0.095 \mathrm{~m} / \mathrm{s})(1)$

Table 1 Clinical and CMR data of the ten patients evaluated by the survey participants

\begin{tabular}{|c|c|c|c|c|c|c|}
\hline Patient & Cardiac diagnosis & $\begin{array}{l}\text { RVEF } \\
\text { (CMR, } \\
\%)\end{array}$ & TAPSE $(\mathrm{mm})$ & $\mathrm{S}^{\prime}(\mathrm{m} / \mathrm{s})$ & FAC $(\%)$ & GLS-RV (\%) \\
\hline 1 & Heart failure with preserved ejection fraction & 74 & 30 & 0.21 & 60 & -40.33 \\
\hline 2 & Coronary artery disease & 71 & 20 & 0.10 & 48 & -25.33 \\
\hline 3 & TTR-amyloidosis, postcapillary pulmonary hypertension & 65 & 17 & 0.10 & 50 & -26.67 \\
\hline 4 & Dilated cardiomyopathy & 58 & 22 & 0.14 & 47 & -24.33 \\
\hline 5 & Sudden cardiac death survivor & 55 & 27 & 0.16 & 60 & -31 \\
\hline 6 & Severe mitral regurgitation, postcapillary pulmonary hypertension & 49 & 16 & 0.11 & 39 & -23 \\
\hline 7 & Idiopathic pulmonary arterial hypertension & 38 & 16 & 0.13 & 27 & -19.33 \\
\hline 8 & Tachy-cardiomyopathy & 38 & 14 & 0.09 & 39 & -16.33 \\
\hline 9 & Combined pre/postcapillary pulmonary hypertension & 34 & 13 & 0.09 & 30 & -14.33 \\
\hline 10 & Idiopathic pulmonary arterial hypertension & 21 & 10 & 0.10 & 22 & -5 \\
\hline
\end{tabular}

$R V E F$ right ventricular ejection fraction, $C M R$ cardiac magnetic resonance imaging, TAPSE tricuspid annular plane systolic excursion, $S^{\prime}$ tissue Doppler imaging basal lateral segment of the free lateral wall of the right ventricle, $F A C$ fractional area change, $G L S-R V$ global longitudinal strain of the free lateral wall of the right ventricle 
Table 2 Demographic and professional characteristics of the study participants $(\mathrm{n}=868)$

\begin{tabular}{|c|c|c|c|}
\hline Characteristics & All & Medical doctors & Sonographers \\
\hline Number of participants, $\mathrm{n}(\%)$ & $868(100)$ & $675(78)$ & $193(22)$ \\
\hline \multicolumn{4}{|l|}{ Age (years) } \\
\hline$<30, \mathrm{n}(\%)$ & $75(9)$ & $48(7)$ & $27(14)$ \\
\hline $30-39$, n $(\%)$ & $309(36)$ & $253(38)$ & $56(29)$ \\
\hline $40-49, \mathrm{n}(\%)$ & $239(27)$ & $188(28)$ & $51(26)$ \\
\hline $50-59$, n $(\%)$ & $156(18)$ & $118(17)$ & $38(20)$ \\
\hline $60-69$, n $(\%)$ & $81(9)$ & $63(9)$ & $18(9)$ \\
\hline$>69, \mathrm{n}(\%)$ & $8(1)$ & $5(1)$ & $3(2)$ \\
\hline \multicolumn{4}{|l|}{ Work setting } \\
\hline University hospital, n (\%) & $251(29)$ & $210(31)$ & $41(21)$ \\
\hline Hospital, n (\%) & 407 (47) & $305(45)$ & $102(53)$ \\
\hline Private practice, n (\%) & $186(21)$ & $143(21)$ & $43(22)$ \\
\hline Other, n (\%) & $24(3)$ & $17(3)$ & $7(4)$ \\
\hline \multicolumn{4}{|l|}{ Level of expertise } \\
\hline Beginner, n (\%) & $144(17)$ & $117(17)$ & $27(14)$ \\
\hline Intermediate, $\mathrm{n}(\%)$ & $410(47)$ & $333(49)$ & $77(40)$ \\
\hline Advanced, n (\%) & $255(29)$ & $183(27)$ & $72(37)$ \\
\hline Expert, n (\%) & $59(7)$ & $42(6)$ & $17(9)$ \\
\hline
\end{tabular}

severely reduced RVEF (RVEF < 30\%). Median RVEF was $52 \%$ (range $21-74 \%$ ). Detailed information on CMR assessment is presented in Table 1.

\section{Statistical analysis}

Categorical data are presented as absolute numbers and percentages. Inter-group comparisons of descriptive data were performed using the $\chi^{2}$ test or Fisher's exact test, as appropriate. A p-value of $<0.05$ was considered statistically significant. All statistical analyses were performed using SPSS Version 24 (IBM SPSS, USA).

First, the ability to detect a decrease in RVF (CMRRVEF $<50 \%$ ) via "eyeballing" alone was evaluated. Sensitivity, specificity, negative predictive value, positive predictive value, area under the curve (AUC), and accuracy were calculated.

Apart from correct classification into normal or decreased function, consensus between a large group of examiners in terms of the degree of dysfunction is necessary to enable uniform nomenclature and reliable follow-up examinations. Therefore, a scoring was performed for concordance between visual assessment of the degree of RV systolic dysfunction and the CMR gold standard ( 0 points, concordant; 1 point, 1 grade difference; 2 points, 2 grade difference; 3 points, 3 grade difference). For instance, if CMR-RVEF was normal with $55 \%$, and the participant graded the RV as mildly reduced, a score of 1 point was attributed. For each participant, the total score for the evaluation of all 10 patients was calculated. The lower the score, the better the agreement between the participant and the CMR diagnosis.
We compared two different systems of classification. A three-grade scale (normal, reduced, severely reduced RVF) and a four-grade scale (normal, mildly reduced, moderately reduced, severely reduced RVF). For the three-grade scale the participants' ratings for mildly and moderately reduced RVF were counted as one combined category (reduced RVF).

\section{Results}

\section{Demographic and professional characteristics of the study participants}

A total of 868 participants completed both the questionnaire and the web-based test. Eighty-one percent of the cohort were between 30 and 60 years of age. A total of $40 \%$ were cardiologists; $22 \%$ were sonographers; and $20 \%$ were internists. Employment settings comprised the following: university hospitals, $29 \%$; non-tertiary hospitals, $47 \%$; and private practice, $21 \%$. The number of participants in each of the four study groups was as follows: $17 \%$, beginners; $47 \%$, intermediate; $29 \%$, advanced; and $7 \%$, expert. The participants were based in 99 different countries, including the following: the United States of America, 11\%; Germany, 8\%; and Austria, 6\%. The demographic and professional characteristics of the participants are shown in Table 2 and Fig. 2. 


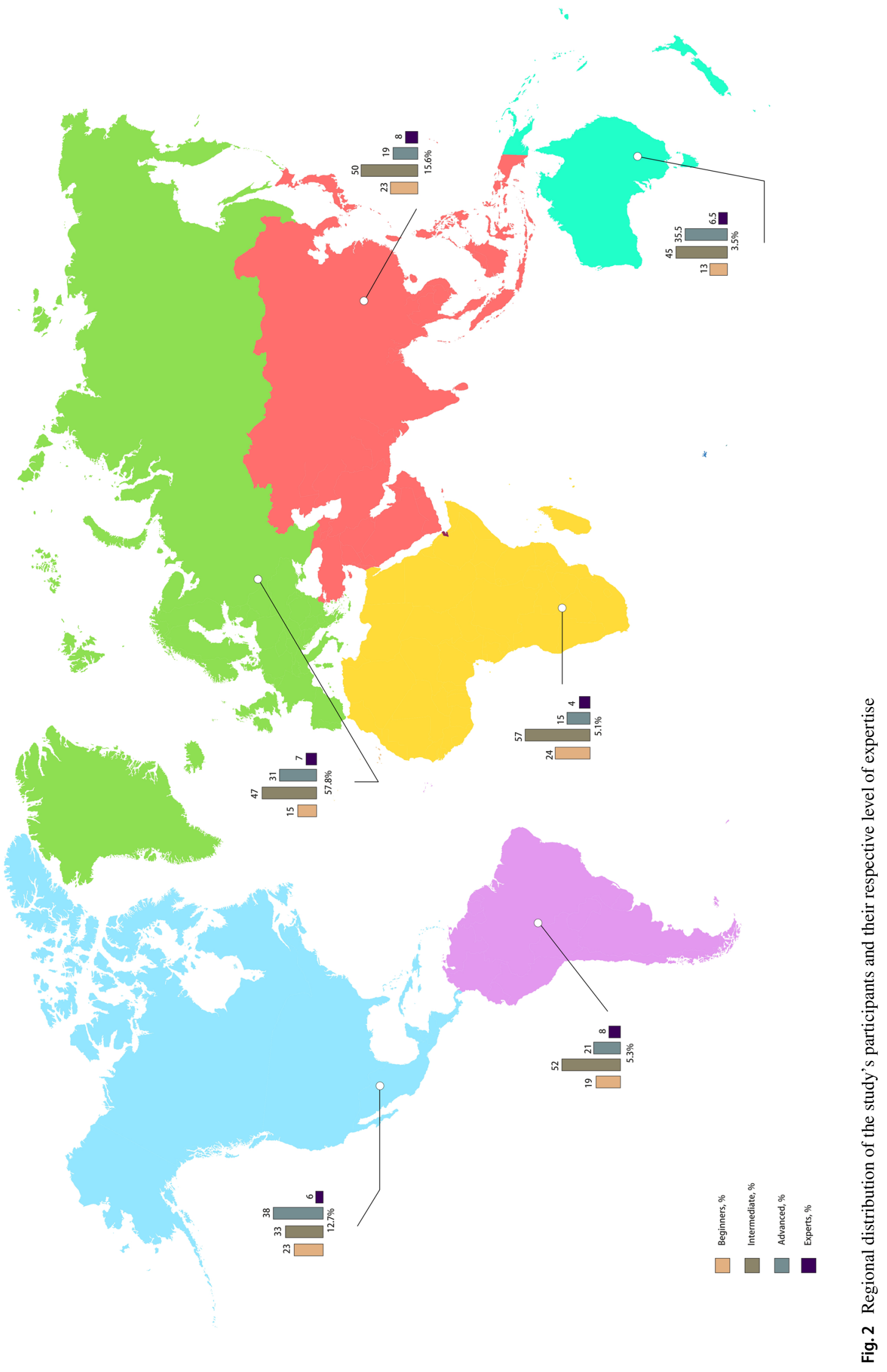


Table 3 Diagnostic accuracy for detection of reduced right ventricular function (mild, moderate, or severe) using CMR-RVEF $>50 \%$ as the gold standard for normal RVF

\begin{tabular}{|c|c|c|c|c|c|}
\hline & Sensitivity (95\% CI) & Specificity $(95 \%$ CI) & PPV & NPV & Accuracy \\
\hline Beginner & $95.8(94.1-97)$ & $42.5(39.1-46)$ & $62.5(61-63.9)$ & $90.9(87.7-93.4)$ & $69.1(66.8-71.4)$ \\
\hline Intermediate & $96.5(95.6-97.3)$ & $54.6(52.4-56.7)$ & $68(66.9-69)$ & $94(92.6-95.2)$ & $75.5(74.2-76.8)$ \\
\hline Advanced & 96.8 (95.7-97.7) & $52.8(50-55.5)$ & $67.2(65.9-68.5)$ & $94.3(92.5-95.8)$ & $74.8(73.1-76.5)$ \\
\hline Experts & $97.1(94.5-98.6)$ & $55.7(50-61.4)$ & $68.7(65.9-71.4)$ & 95 (90.8-97.3) & $76.4(72.8-79.7)$ \\
\hline
\end{tabular}

$C M R$ cardiac magnetic resonance imaging, $R V F$ right ventricular function, $P P V$ positive predictive value, $N P V$ negative predictive value

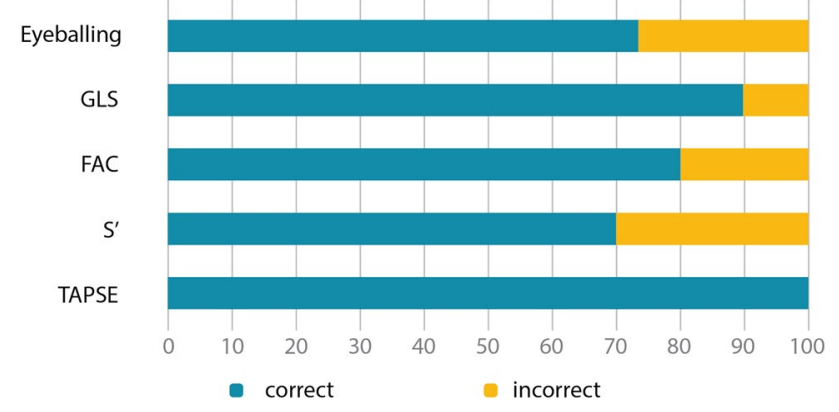

Fig. 3 Correct identification of reduced right ventricular function (RVF, defined as cardiac magnetic resonance imaging derived right ventricular ejection fraction $<50 \%$ ) of the different methods of RVF gradation. $G L S$ global longitudinal strain, $F A C$ fractional area change, $S$ ' tissue Doppler imaging basal free lateral wall of the right ventricle, TAPSE tricuspid annular plane systolic excursion

\section{Detection of reduced RVF (CMR-RVEF $<50 \%)$}

Correct detection of reduced RVF (CMR-derived RVEF $<50 \%$ ) was analyzed for each of the four study groups. Sensitivity was $95.8 \%, 96.5 \%, 96,8 \%$, and $97.1 \%$ for the beginner, intermediate, advanced, and expert groups, respectively. Specificity in these four groups was $42.5 \%, 54.6 \%, 52.8 \%$, and $55.7 \%$, respectively. Better concordance was found for the intermediate, advanced, and expert groups than for beginners (Table 3). When beginners were compared with the combined advanced, moderately advanced, and expert groups, the area under the curve (AUC) was 0.69 for beginners vs. 0.75 in the combined non-beginners group.

Correct identification of reduced RVF was compared between "eyeballing" and echocardiographic parameters. While the study participants differentiated correctly in $74 \%$ of cases by "eyeballing", correct differentiation was found for TAPSE in all 10 patients, GLS-RV in nine patients, FAC in eight patients, and $S^{\prime}$ in seven patients (Fig. 3).

\section{Determination of the degree of RV systolic dysfunction}

Two different systems of systolic function gradation were compared. For the four-grade system, $8 \%$ of the participants

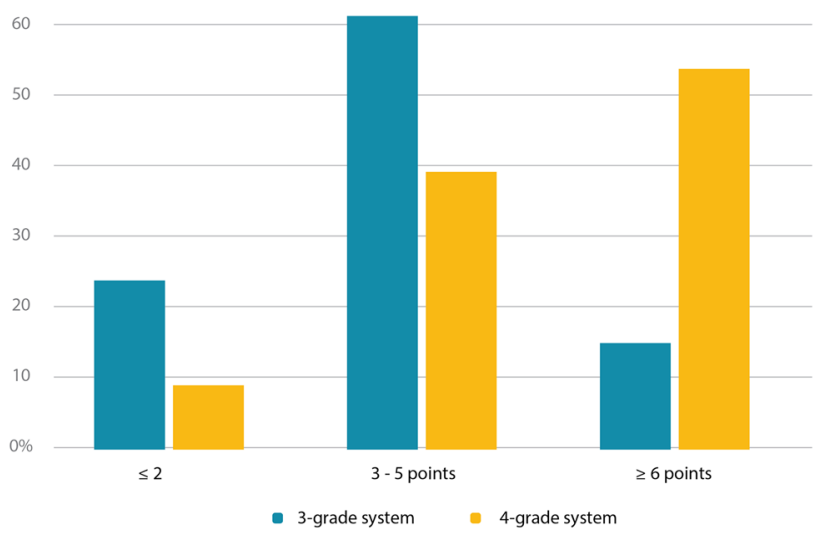

Fig. 4 Comparison of 3-grade system (normal, reduced, severely reduced) against 4-grade system (normal, mildly reduced, moderately reduced, severely reduced). $\leq 2$ points indicate excellent concordance with $C M R, \geq 6$ points indicate poor concordance with $C M R$. $C M R$ cardiac magnetic resonance imaging

scored $\leq 2$ points, indicating very good concordance with CMR, and $54 \%$ of the participants scored $\geq 6$ points, indicating poor concordance with CMR. In the three-grade system, $23 \%$ of the participants scored $\leq 2$ points, and $14 \%$ of the participants scored $\geq 6$ points (Fig. 4).

\section{Accuracy in determining the degree of RV systolic dysfunction}

Further analysis was then performed for the three-grade system. Participants from the expert, advanced, and intermediate groups assigned a diagnosis that was concordant with CMR in 56\%, 56\%, and 57\% of cases, respectively. Participants from these groups assigned a level that was one grade different to CMR in $43 \%, 43 \%$, and $42 \%$ of cases, respectively, and two grades different to CMR in $0.2 \%, 1 \%$, and $1 \%$ of cases, respectively. Participants from the beginners group were concordant with CMR in $48 \%$ of cases, and assigned a level that was one grade different to CMR in $48 \%$ of cases, and two grades different to CMR in $4 \%$ of cases (Fig. 5). Less than $10 \%$ of the 868 participants assigned the correct diagnosis in $100 \%$ of cases. A total of $80 \%$ or more correct diagnoses were assigned by the following percentages of 


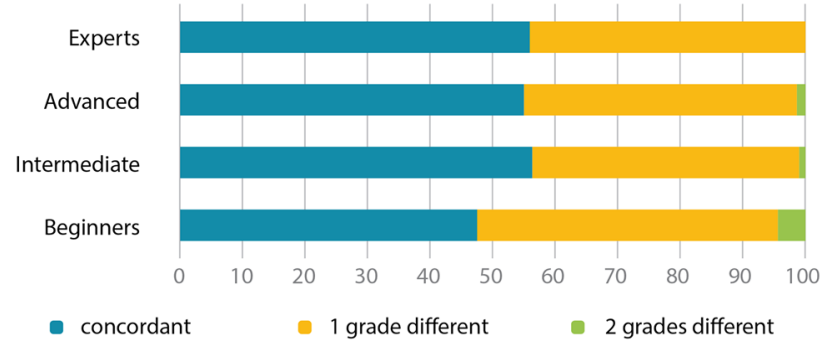

Fig. 5 Concordance with cardiac magnet resonance imaging derived right ventricular ejection fraction of the different levels of expertise

individuals from each study group: $33 \%$, beginners; $51 \%$, intermediate; $47 \%$, advanced; and 55\%, expert. Compared with the other three groups, significantly fewer correct diagnoses, and more incorrect diagnoses, were observed in the beginners group $(\mathrm{p}<0.001)$.

\section{Discussion}

The present investigation of "eyeballing" alone for the evaluation of RVF showed that, while sensitivity for the detection of reduced RVF was excellent in all expertise levels, including beginners, specificity was poor. In addition, determination of the degree of RV systolic dysfunction was imprecise.

To our knowledge, the present study is the first to utilize the potential of the internet in order to investigate this issue in an international context. A major strength of the analyses was the large study cohort, which represented a broad range of echocardiography expertise and clinical settings.

\section{Detection of reduced RVF (CMR-RVEF $<50 \%)$}

Evaluation of the ability to detect reduced RVF revealed excellent sensitivity and negative predictive value in all four study groups. The more experienced the examiner, the better were sensitivity, specificity, and accuracy. Differences between the groups were marginal, though. Even beginners identified reduced RVF in most cases. This is reassuring, since reduced RVF is a crucial finding that must prompt further diagnostic workup. For instance, reduced RVF impacts the management of pulmonary embolism. In these patients, echocardiography is frequently performed by inexperienced non-cardiologists, and occasionally with the use of wireless handheld devices, for example, in the emergency medicine setting. In these cases, measurement of more sophisticated RVF parameters is precluded. The present data indicate that echocardiography is a very useful tool, also for noncardiologists in non-tertiary settings. Thus, in view of the ever decreasing cost and increased availability of ultrasound machines, this suggests that basic medical education should involve expanded training in basic echocardiography.
Specificity was very poor in all four study groups, with the highest specificity being found among experts and the lowest among beginners. The low specificity observed in the present analyses may have been attributable to the fear of overlooking patients with reduced RVF.

Analyses were also performed to determine the degree to which reduced RVF could be detected using more sophisticated echocardiography parameters. TAPSE reliably distinguished between normal and reduced RVF in all 10 patients, while both FAC and GLS-RV were more accurate than "eyeballing" alone. Therefore, comprehensive evaluation of RVF should include a range of parameters to correct for potential visual errors. In cases of diagnostic doubt, the present data suggest that an experienced examiner should re-evaluate the acquired images, and that other imaging modalities, such as CMR, should be considered.

\section{Accuracy of the determination of the degree of RV systolic dysfunction}

Across all four study groups, significant disagreement was apparent, in particular with respect to mildly and moderately reduced RVF. For this reason, accuracy with the three-grade scale was superior. If diagnosis is based solely on visual assessment and 2D echo parameters such as TAPSE, S', FAC, and GLS-RV, the present authors propose the use of a three-grade system for determination of the degree of RV systolic dysfunction. With 3D echocardiography, an additional parameter has become available in recent years. If a reliable 3D echocardiography dataset is available, RVEF can be calculated, thus allowing further classification into mildly and moderately reduced RVF.

When applying the three-grade system, even in the expert group, only half of the examiners classified $80 \%$ or more of the patients in accordance with gold standard CMR values. While beginners had slightly lower rates of concordant diagnoses, similar results were found across the expert, advanced, and intermediate groups. These findings suggest that this skill can be learned quickly and is performed on a similar, albeit unsatisfactory, level across all levels of expertise beyond novice status.

\section{Limitations}

The present study had limitations. First, participants were recruited from the users of an online teaching platform, which may have introduced a selection bias. This selection bias may have led to the inclusion of professionals with an interest in continued medical education and an open attitude towards new technologies. Level of expertise was selfreported, allowing for false classification both to more and 
to less advanced levels than accurate. Second, evaluation of RVF was based solely on a RV-focused four-chamber view. In clinical practice, RVF should always be evaluated using several angulations of the RV. However, the one-loop questions enabled a very low threshold for participation and thus a large study cohort. Third, in the real-world setting, assessment of RVF is also dependent on the ability to acquire good images. All video loops in the present study had good image quality. This precludes generalizability of the study. Fourth, as suggested by the literature, CMRRVEF was used as the gold standard for RVF. Ejection fraction describes volume change during the cardiac cycle. In the RV, a close relationship exists between myocardial contraction, pulmonary pressure, and the pulmonary vasculature, which is not accounted for by RVEF. Future research is necessary to determine whether superior parameters for gold standard RVF measurement are available, which might correlate better with clinical status and outcome. Finally, CMR and echo were not acquired simultaneously. However, the mean time interval between the two examinations was only 12 days, and all patients were in stable clinical condition with no relevant volume shifts having been recorded between the two assessments.

\section{Conclusion}

To our knowledge, the present study is the first international investigation to demonstrate that "eyeballing" alone differentiates between normal and abnormal RVF with excellent sensitivity but poor specificity, and that accurate classification of the degree of RV systolic dysfunction via "eyeballing" alone is imprecise, even among expert echocardiographers. Better concordance with the gold standard was found for the three advanced groups as compared with beginners. However, overall assessment quality was unsatisfactory. In accordance with current guidelines, the present data suggest that "eyeballing" should be combined with measurement of other parameters of RVF.

Acknowledgements Open access funding provided by Medical University of Vienna.

\section{Compliance with ethical standards}

Conflict of interest All authors declare that they have no conflict of interest.

Ethical approval The study protocol conformed to the ethical guidelines of the Declaration of Helsinki.

Open Access This article is distributed under the terms of the Creative Commons Attribution 4.0 International License (http://creativeco mmons.org/licenses/by/4.0/), which permits unrestricted use, distribution, and reproduction in any medium, provided you give appropriate credit to the original author(s) and the source, provide a link to the Creative Commons license, and indicate if changes were made.

\section{References}

1. Gudmundsson P, Rydberg E, Winter R, Willenheimer R (2005) Visually estimated left ventricular ejection fraction by echocardiography is closely correlated with formal quantitative methods. Int J Cardiol. 101(2):209-212

2. Shahgaldi K, Gudmundsson P, Manouras A, Brodin LA, Winter R (2009) Visually estimated ejection fraction by two dimensional and triplane echocardiography is closely correlated with quantitative ejection fraction by real-time three dimensional echocardiography. Cardiovasc Ultrasound. 7:41 (quiz 86-8)

3. Rudski LG, Lai WW, Afilalo J, Hua L, Handschumacher MD, Chandrasekaran K et al (2010) Guidelines for the echocardiographic assessment of the right heart in adults: a report from the American Society of Echocardiography endorsed by the European Association of Echocardiography, a registered branch of the European Society of Cardiology, and the Canadian Society of Echocardiography. J Am Soc Echocardiogr. 23(7):685-713

4. Lang RM, Badano LP, Mor-Avi V, Afilalo J, Armstrong A, Ernande L et al (2015) Recommendations for cardiac chamber quantification by echocardiography in adults: an update from the American Society of Echocardiography and the European Association of Cardiovascular Imaging. Eur Heart J Cardiovasc Imaging. 16(3):233-270

5. Pavlicek M, Wahl A, Rutz T, de Marchi SF, Hille R, Wustmann K et al (2011) Right ventricular systolic function assessment: rank of echocardiographic methods vs cardiac magnetic resonance imaging. Eur J Echocardiogr. 12(11):871-880

6. Schneider M, Aschauer S, Mascherbauer J, Ran H, Binder C, Lang I et al (2018) Echocardiographic assessment of right ventricular function: current clinical practice. Int J Cardiovasc Imaging. 35(1):49-56

7. Bellsham-Revell HR, Simpson JM, Miller OI, Bell AJ (2013) Subjective evaluation of right ventricular systolic function in hypoplastic left heart syndrome: how accurate is it? J Am Soc Echocardiogr. 26(1):52-56

8. Ling LF, Obuchowski NA, Rodriguez L, Popovic Z, Kwon D, Marwick TH (2012) Accuracy and interobserver concordance of echocardiographic assessment of right ventricular size and systolic function: a quality control exercise. J Am Soc Echocardiogr. 25(7):709-713

9. Magunia H, Schmid E, Hilberath JN, Häberle L, Grasshoff C, Schlensak C et al (2017) 2D Echocardiographic evaluation of right ventricular function correlates with 3D volumetric models in cardiac surgery patients. J Cardiothorac Vasc Anesth. 31(2):595-601

10. Schulz-Menger J, Bluemke DA, Bremerich J, Flamm SD, Fogel MA, Friedrich MG et al (2013) Standardized image interpretation and post processing in cardiovascular magnetic resonance: Society for Cardiovascular Magnetic Resonance (SCMR) board of trustees task force on standardized post processing. J Cardiovasc Magn Reson. 15:35

11. Kawel-Boehm N, Maceira A, Valsangiacomo-Buechel ER, VogelClaussen J, Turkbey EB, Williams R et al (2015) Normal values for cardiovascular magnetic resonance in adults and children. $\mathrm{J}$ Cardiovasc Magn Reson. 17:29

Publisher's Note Springer Nature remains neutral with regard to jurisdictional claims in published maps and institutional affiliations. 